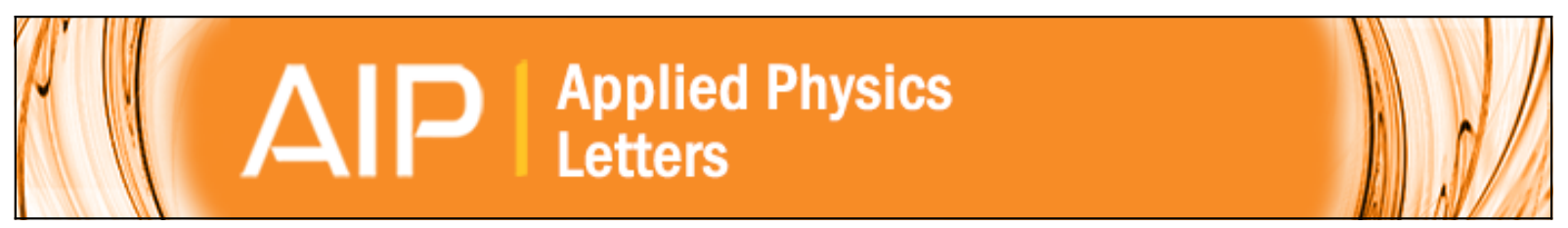

\title{
Near-field mapping of Fano resonances in all-dielectric oligomers
}

Dmitry S. Filonov, Alexey P. Slobozhanyuk, Alexander E. Krasnok, Pavel A. Belov, Elizaveta A. Nenasheva, Ben Hopkins, Andrey E. Miroshnichenko, and Yuri S. Kivshar

Citation: Applied Physics Letters 104, 021104 (2014); doi: 10.1063/1.4858969

View online: http://dx.doi.org/10.1063/1.4858969

View Table of Contents: http://scitation.aip.org/content/aip/journal/apl/104/2?ver=pdfcov

Published by the AIP Publishing

\section{Articles you may be interested in}

Magnetic moment enhancement and spin polarization switch of the manganese phthalocyanine molecule on an IrMn(100) surface

J. Chem. Phys. 141, 034703 (2014); 10.1063/1.4889934

The Fano-type transmission and field enhancement in heterostructures composed of epsilon-near-zero materials and truncated photonic crystals

Appl. Phys. Lett. 103, 201902 (2013); 10.1063/1.4829858

Tunable two types of Fano resonances in metal-dielectric core-shell nanoparticle clusters

Appl. Phys. Lett. 103, 111115 (2013); 10.1063/1.4821187

Experimental realization of all-dielectric composite cubes/rods left-handed metamaterial J. Appl. Phys. 109, 084918 (2011); 10.1063/1.3575326

$\mathrm{Ab}$ initio studies of ferromagnetic properties of a nitronyl nitroxide radical and a nucleoside with -conjugated nitroxide spin label

Appl. Phys. Lett. 90, 182502 (2007); 10.1063/1.2732830

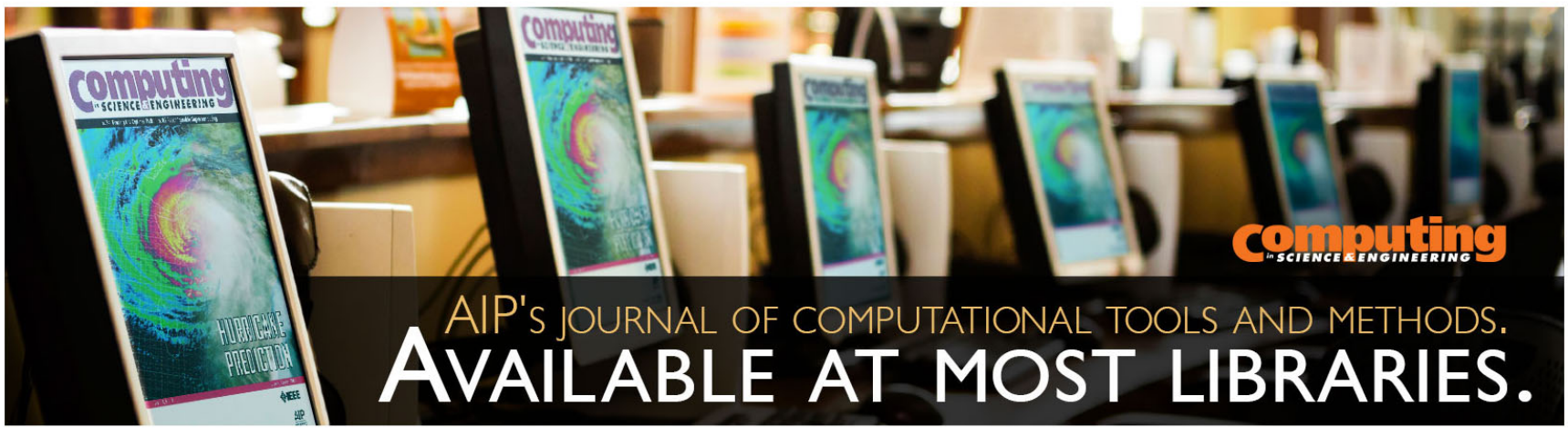




\title{
Near-field mapping of Fano resonances in all-dielectric oligomers
}

\author{
Dmitry S. Filonov, ${ }^{1}$ Alexey P. Slobozhanyuk, ${ }^{1}$ Alexander E. Krasnok, ${ }^{1}$ Pavel A. Belov, ${ }^{1}$ \\ Elizaveta A. Nenasheva, ${ }^{2}$ Ben Hopkins, ${ }^{3}$ Andrey E. Miroshnichenko, ${ }^{3}$ and Yuri S. Kivshar ${ }^{1,3}$ \\ ${ }^{1}$ National Research University of Information Technologies, Mechanics and Optics (ITMO), \\ St. Petersburg 197101, Russia \\ ${ }^{2}$ GIRICOND Research Institute, Ceramics Co. Ltd., St. Petersburg 194223, Russia \\ ${ }^{3}$ Nonlinear Physics Center, Research School of Physics and Engineering, Australian National University, \\ Canberra ACT 0200, Australia
}

(Received 14 November 2013; accepted 13 December 2013; published online 13 January 2014)

\begin{abstract}
We demonstrate experimentally Fano resonances in all-dielectric oligomers clusters of dielectric particles. We study two structures consisting of a ring of six ceramic spheres with and without a central particle and demonstrate that both structures exhibit resonant suppression of the forward scattering associated with the Fano resonance originated from the excitation of magnetic dipole modes. By employing the near-field measurement techniques, we establish the relation between near- and far-field properties of the Fano resonances and identify directly their origin. We support our findings by an analytical approach based on the discrete-dipole approximation and find an excellent agreement with the experimental data. (C) 2014 AIP Publishing LLC.

[http://dx.doi.org/10.1063/1.4858969]
\end{abstract}

The physics of Fano resonance originates from wave interference, and it is usually associated with interference of broad (nonresonant) and narrow (resonant) spectra resulting in characteristic asymmetric profiles. The asymmetry of the Fano-resonance lineshape is due to the fact that both destructive and constructive interference phenomena take places in the narrow spectral range. ${ }^{1}$

Fano resonance has been observed in various branches of physics including photonics, plasmonics, and metamaterials. $^{2}$ Recently, much effort has been devoted to the study of Fano resonances in the so-called plasmonic oligomer structures consisting of a finite number of metallic nanoparticles arranged in a symmetric fashion. ${ }^{3-11}$ The Fano resonance manifests itself as a resonant suppression of the total scattering cross-section accompanied by an enhanced absorption. This suppression can be understood as being a result of destructive interference of two excited plasmonic modes of the structure, one of them being at resonance. One of the main issues in the physics of Fano resonances is to identify correctly the interfering modes, especially in complex structures of many particles. A difficulty comes from the fact that usually we are dealing with supermodes of interacting nanoparticles, and those modes crucially depend on the particles arrangement and their geometry. There exist many theoretical approaches, suggesting how to identify the modes which contribute to interference in the vicinity of the Fano resonances, e.g., plasmonic hybridization approach, ${ }^{3,12-14}$ symmetry-adapted linear combinations of dipole moments, ${ }^{3,4,9,15}$ and others.

Experimentally, real-space mapping of Fano interference in plasmonic oligomers has been suggested to identify intensity and phase of separate metallic nanoparticles, for various geometric sizes and the illumination wavelength. ${ }^{7,16}$ All these approaches decompose rather complex near-field profiles of plasmonic oligomers onto a superposition of resonant (narrow) and nonresonant (broad) states. Although these methods do provide a useful insight into the origin of Fano resonances in plasmonic oligomers, they still do not provide a complete picture, since they are not able to predict a priori if a certain structure would exhibit a Fano resonance.

Recently, we have predicted theoretically that alldielectric oligomers, symmetric clusters of dielectric particles, do possess Fano resonances. ${ }^{17}$ The main feature of the dielectric oligomers, in comparison with their metallic counterparts, is that the electromagnetic field is localized inside the dielectric particles. Another important property is that the lower mode of a spherical high-refractive index dielectric particle is a magnetic dipole mode ${ }^{18,19}$ recently observed experimentally in microwave ${ }^{20}$ and visible regions. ${ }^{21,22}$ The origin of this magnetic response is due to the excitation of a circulating displacement current. It takes place when a particle's diameter becomes comparable with the wavelength inside the particle. Thus, the resonant wavelength of the magnetic mode scales linearly with the size. ${ }^{21,23,24}$ It provides the great advantage of tuning the resonant response of nanophotonic structures involving dielectric particles. ${ }^{25}$

Similar to the theory of plasmonic oligomers, we expect that a heptamer structure consisting of six identical dielectric particles located at the corners of a hexagon and a particle of a different radius in the center, would demonstrate the Fano resonance at the resonant frequency of the central particle. ${ }^{17}$ Indeed, due to a strong field localization inside dielectric particles, the resonant supermodes can be easily identified in this case. One mode is associated with the field localized around the central particle, whereas other six particles of different radii will be out of resonance, and they will form a broad supermode. The near-field interference of these two modes leads to the suppression of the total scattering associated with the Fano resonances. ${ }^{17}$

In this Letter, we confirm experimentally the existence of the Fano resonances in dielectric oligomer structures. Using the scalability of Maxwell's equations, we use macroscopic ceramic spherical particles of the centimeter scale (instead of silicon nanoparticles) which exhibit a similar 


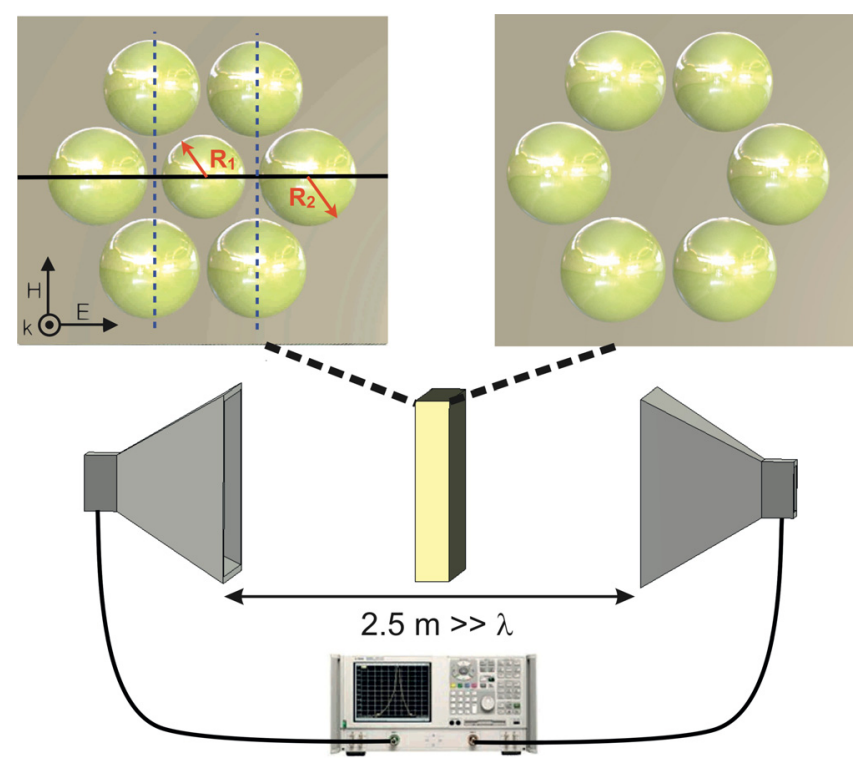

FIG. 1. Schematic view of the experimental setup and images of two structures under study (a) heptamer and (b) hexamer dielectric oligomers.

response in the microwave region. The advantage of using such large dielectric spheres is the ability to measure nearfield profiles with a high precision, including both phase and intensity. It allows us to establish the relation between near- and far-field properties in the vicinity of the Fano resonances and identify their origins. We notice that the total scattering cross-section of oligomer-like structures is polarization independent, ${ }^{26}$ and so is the Fano resonance, which allows to simplify the experimental setup. To support our findings, we employ the analytical approach based on the discrete-dipole approximation and observe an excellent agreement with the experimental data.

To mimic the scattering properties of silicon nanoparticles in the microwave frequency range, we use $\mathrm{MgO}-\mathrm{TiO}_{2}$ ceramic spheres characterized by similar dielectric constant of 16 and dielectric loss factor of $(1.12-1.17) \times 10^{-4}$, measured at $9-12 \mathrm{GHz}$ frequency range.$^{27}$ In Fig. 1, we show an experimental setup with two types of dielectric oligomers. The heptamer oligomer consists of a central dielectric sphere of radius $R_{1}=6 \mathrm{~mm}$ and a ring of dielectric spheres with radius $\mathrm{R}_{2}=7.5 \mathrm{~mm}$. Distance between the centers of the particles is $15.9 \mathrm{~mm}$. To fasten together the particles, we use a special holder made of a styrofoam material with dielectric permittivity of 1 (in the microwave frequency range). To approximate the plane wave excitation, we employ a rectangular horn antenna (TRIM $0.75-18 \mathrm{GHz}$; DR) connected to the transmitting port of a vector network analyzer (Agilent E8362C). The oligomer is located in the far-field of the antenna (the distance approximately $2.5 \mathrm{~m}$ ), and the second
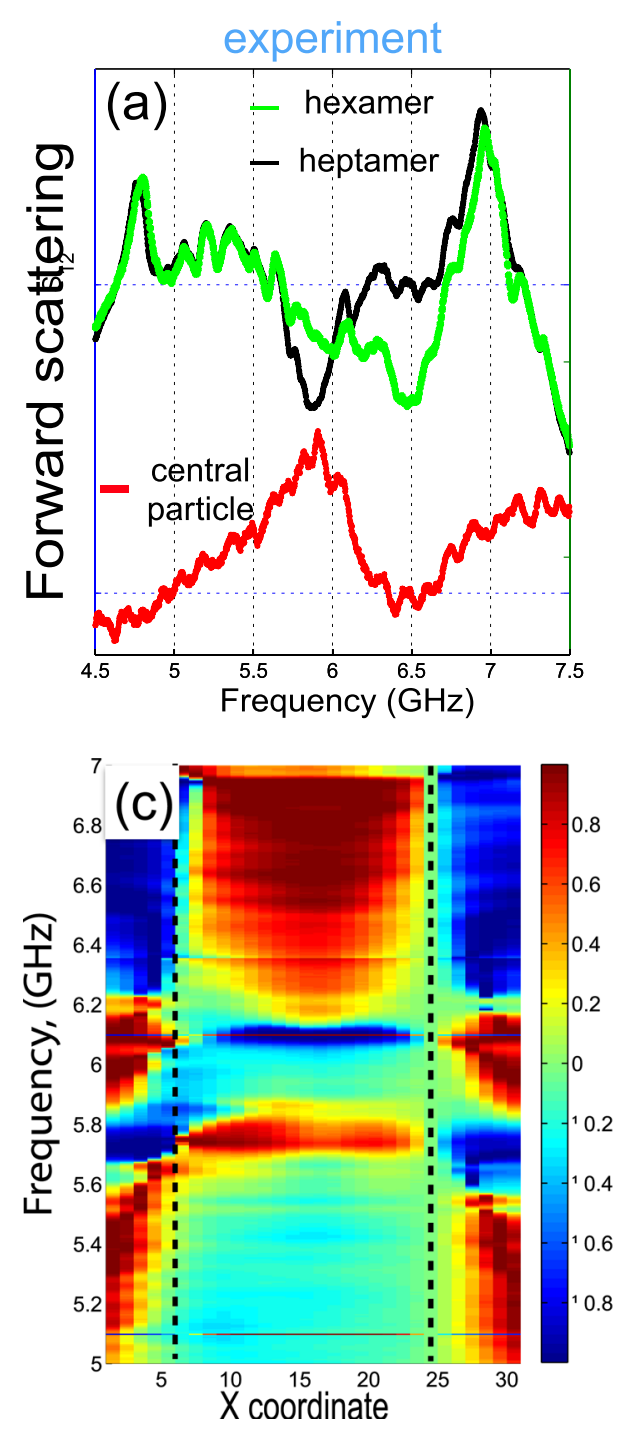
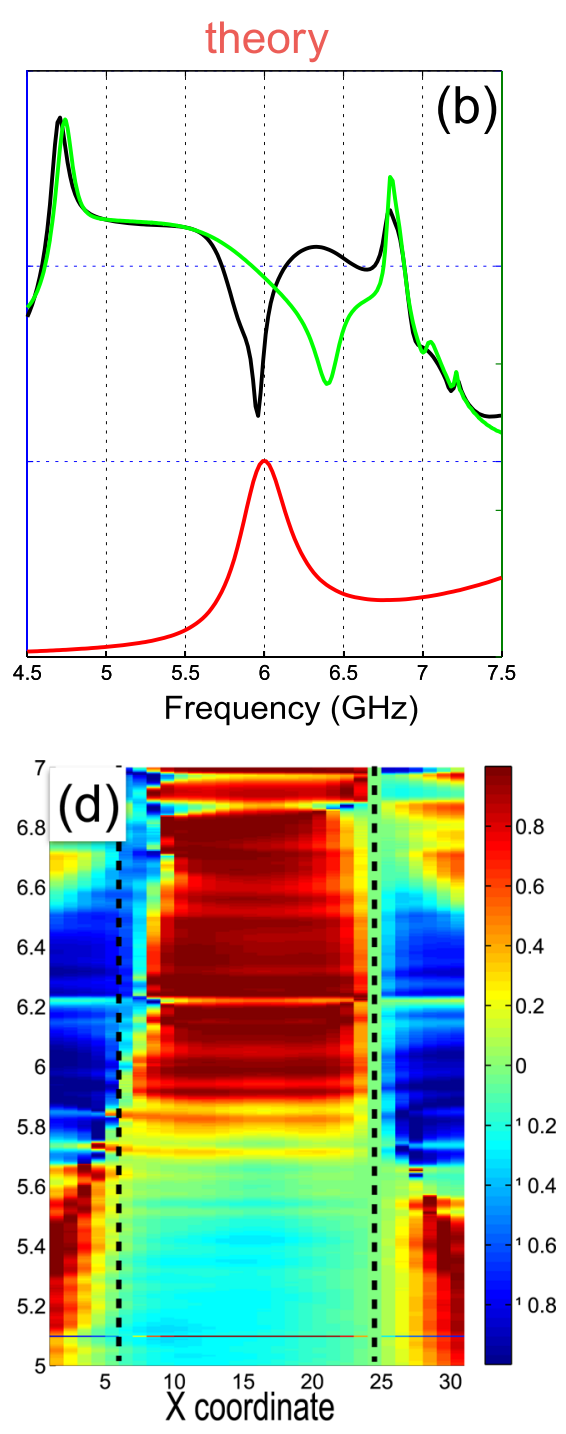

FIG. 2. (a) Experimentally measured and (b) numerically calculated forward scattering of a single central particle (red), hexamer (green), and heptamer (black) oligomers. Phase map of the magnetic field along the central line of (c) heptamer and (d) hexamer dielectric oligomers. 
horn antenna (TRIM $0.75-18 \mathrm{GHz}$; DR) is used as a receiver. The experimentally measured and numerically calculated forward scattering of a single central particle (red line), a hexamer (green line), and a heptamer (black line) are shown in Figs. 2(a) and 2(b).

In Figs. 2(a) and 2(b), we observe that the heptamer structure exhibits a resonant suppression of the forward scattering around $6 \mathrm{GHz}$. In accordance with the optical theorem, scattering in the forward direction is connected with the total extinction cross-section. Since absorption by dielectric particles is very weak, the total extinction cross-section is equal to the scattering cross-section, and therefore, we arrive at the conclusion that such a resonant suppression should be also observed in the total scattering cross-section.

To answer the question if this scattering suppression is related to the Fano resonance, we should identify at least two dissimilar modes which can interfere with each other. The resonant mode in this case can be easily identified, and it is attributed to the magnetic resonance of the central particle [see Figs. 2(a) and 2(b)], as was predicted theoretically. ${ }^{17}$ The nonresonant mode is created by a collective response of the ring of six particles. Since the particle's radius is larger and the magnetic resonance depends linearly on the size, this resonance is red shifted with respect to that of the central particle. Thus, in the case of heptamer oligomers, we can always predict that the Fano resonance takes place near the resonant frequency of the central particle.

To justify this physical picture of the mode interference, next we employ the coupled-dipole approximation to identify precisely the critical modes contributing to the Fano resonance. We employ the method suggested in Ref. 28 and represent each dielectric particle by a pair of electric and magnetic dipoles with proper polarizabilities derived from the exact Mie solutions. As a result (for details, see Ref. 29), the system of coupled dipoles can be described by the corresponding interaction matrix, whose eigenvectors give the profiles of supermodes of the structure. By decomposing the final solution into these modes, we extract the information about excited collective modes. The results of this analysis are presented in Fig. 3. Following some symmetry arguments, ${ }^{29}$ we can prove that there exist only three independent supermodes of the heptamer oligomer that can be excited by the linearly polarized plane wave. From Figs. 3(c) and 3(e), we observe that the resonance suppression of the forward scattering takes place at the crossing of two out-of-phase magnetic supermodes. As is expected, one of these magnetic modes is localized around the central particle [see Fig. 3(e), green] supporting directly our qualitative analysis above and earlier theoretical predictions. ${ }^{17}$ Moreover, this supermode is enhanced at the magnetic resonance of the central particle, where its effective magnetic dipole exhibits the $\pi$-shift being out-of-phase the ring mode, leading subsequently to the Fano-like destructive interference.

To support these results experimentally, we perform the near-field scanning for the frequencies within the $5-7 \mathrm{GHz}$ frequency band. We use an automatic mechanical near-field scanning device and a shielded loop probe connected to the receiving port of the Analyzer. The shielded loop had a
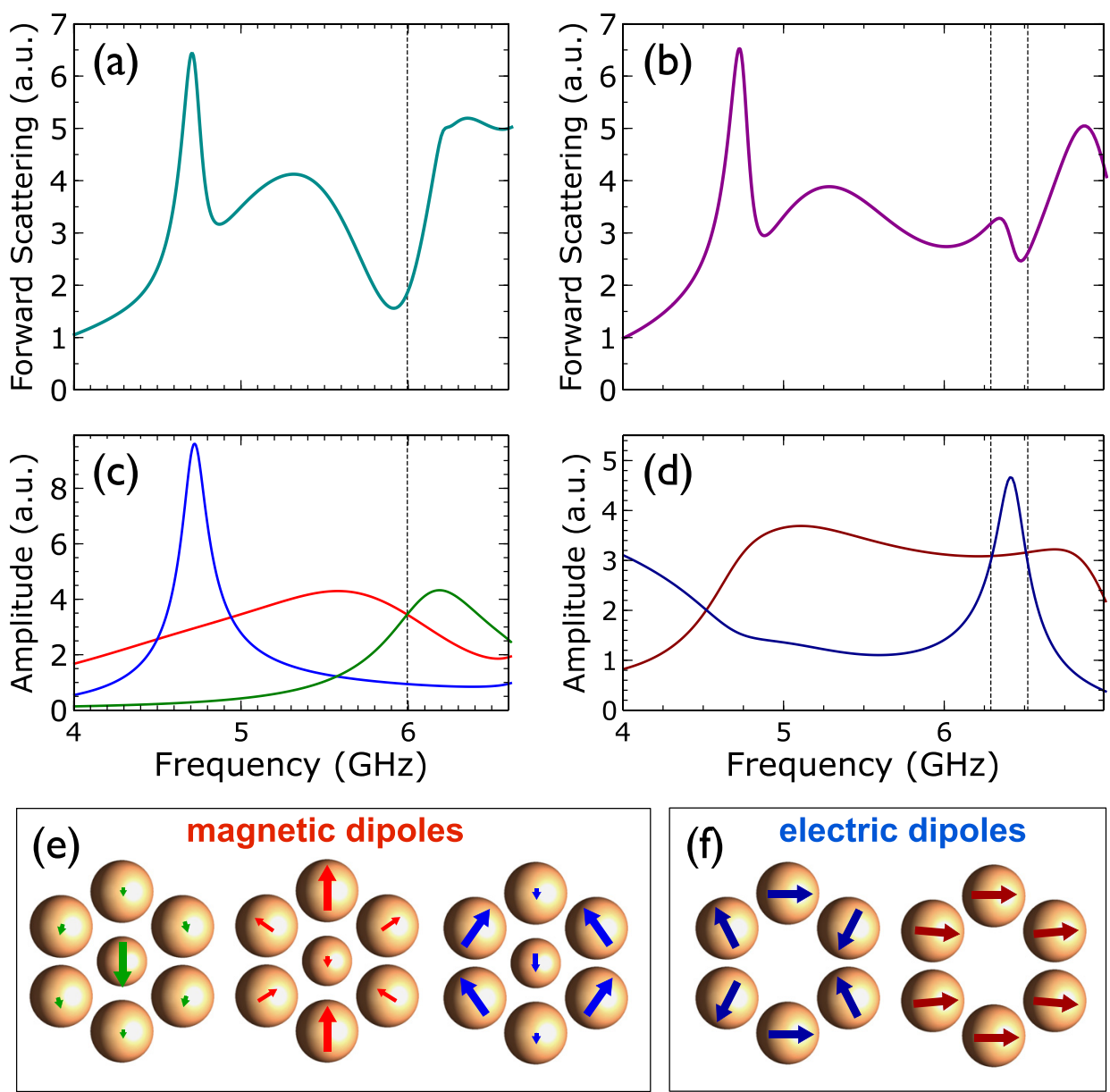
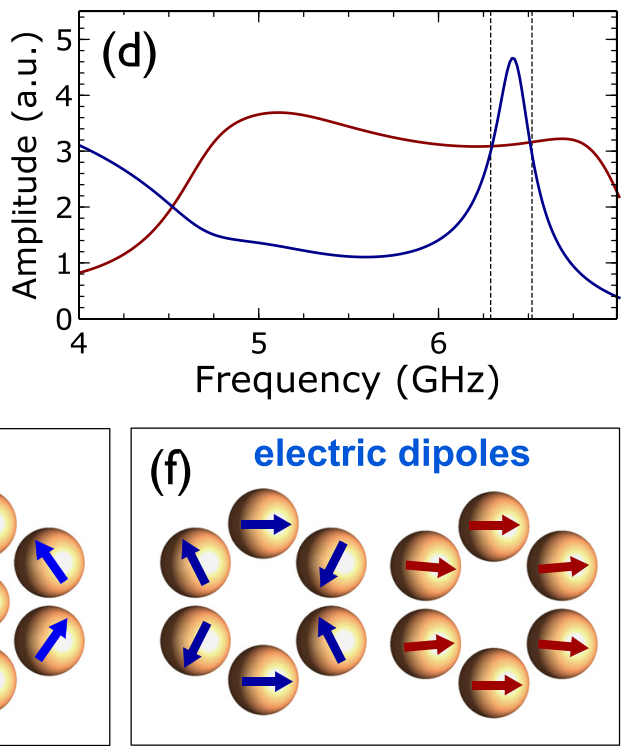

FIG. 3. Calculated forward scattering for (a) heptamer and (b) hexamer oligomers. Panels (c) and (d) show the corresponding projection of the total solution onto supermodes. Profiles of the excited supermodes at the Fano resonance are shown in panels (e) and (f) for heptamer and hexamer oligomers, respectively. 
$6 \mathrm{~mm}$ diameter, and it could be approximated as an electrically small current loop that measures the magnetic field in the forward direction, without significant field averaging. The probe was oriented normally with respect to the interfaces of both oligomer and transmission horn antenna (measured dominantly $H$-component of the horn). The near-field mapping is scanned at the distance of $2 \mathrm{~mm}$ away from the back interface of the oligomer, in order to avoid touching of the probe and sample. In order to demonstrate the existence of the Fano resonance in all-dielectric oligomer, we measure the phase with the dependence on the coordinate along the black line in Fig. 1 as well as the frequency. The phase jump up to $\pi$ in the heptamer structure can be clearly observed in Fig. 2(c), and it confirms that the induced magnetic moments of the central and ring dielectric particles are out of phase, which corresponds to the physics of the Fano resonance.

Another interesting point is the suppression of the forward scattering for the hexamer oligomer. In this case, we have only six identical particles placed on the ring without a central particle. Remarkably, there exists no associated $\pi$ phase jump of the magnetic near-field around the resonant frequency at $6.5 \mathrm{GHz}$ [see Fig. 2(d)]. Therefore, a natural question is, if such a structure may also support a Fano resonance, where do the modes and destructive interference come from? To answer this question, we employ again the coupleddipole approach developed in Ref. 29. By decomposing the final solution into supermodes of the hexamer, we find that there exist only two electric-type modes, which contribute to the Fano lineshape in the overall extinction [see Figs. 3(b), 3(d), and 3(f)]. A principal difference between this case and the case of heptamer is that the electric-type supermodes of the hexamer are dominant, due to proximity to the electric dipole resonance of individual dielectric particles. Thus, in the vicinity of the resonant suppression of the forward scattering at $6.5 \mathrm{GHz}$, one supermode is resonantly enhanced [see Fig. 3(d)]. We also notice that now there appear two intersection points of those modes at $6.4 \mathrm{GHz}$ and $6.6 \mathrm{GHz}$, respectively. One of them corresponds to destructive and the other to constructive interference phenomena. As a result, the scattering profile will be associated with the generic and fully asymmetric Fano resonance.

In conclusion, we have demonstrated experimentally the existence of Fano resonances in all-dielectric oligomer structures. We have verified the earlier theoretical prediction that Fano resonances in heptamer structures are associated with the magnetic dipole resonance of the central particle. We have confirmed this result by both far- and near-field measurements, including the studies of the forward scattering and phase distribution across the structure. The resonant suppression of the scattering was observed to be accompanied by the $\pi$ phase jump at the central particle. We have performed the modal analysis based on the coupled-dipole approximation, which allows to clearly identify the particular supermodes contributing to the resonant interference phenomena. In addition, we have also observed a unique type of Fano resonance in hexamer oligomers without a central particle. By employing the modal analysis, we were able to identify that this type of resonance originates from the interference of electric-type supermodes, and it exhibits an asymmetric profile due to the presence of both constructive and destructive interference phenomena.

This work was supported by the Ministry of Education and Science of the Russian Federation (Project Nos. 11.G34.31.0020, 11.519.11.2037, 14.B37.21.0307, and 01201259765), the Russian Foundation for Basic Research (Nos. 12-02-12097, 13.02.00411, 13.02.00623, 13.02.01331, and 14.02.31268), Dynasty Foundation (Russia), and the Australian Research Council.

${ }^{1}$ A. E. Miroshnichenko, S. Flach, and Y. S. Kivshar, Rev. Mod. Phys. 82, 2257 (2010).

${ }^{2}$ B. Luk'yanchuk, N. I. Zheludev, S. A. Maier, N. J. Halas, P. Nordlander, H. Giessen, and C. T. Chong, Nature Mater. 9, 707 (2010).

${ }^{3}$ N. A. Mirin, K. Bao, and P. Nordlander, J. Phys. Chem. A 113, 4028 (2009).

${ }^{4}$ N. Verellen, Y. Sonnefraud, H. Sobhani, F. Hao, V. V. Moshchalkov, P. V. Dorpe, P. Nordlander, and S. A. Maier, Nano Lett. 9, 1663 (2009).

${ }^{5}$ Mario Hentschel, Michael Saliba, Ralf Vogelgesang, Harald Giessen, A. Paul Alivisatos, and Na Liu, Nano Letters 10(7), 2721-2726 (2010).

${ }^{6}$ K. Bao, N. Mirin, and P. Nordlander, Appl. Phys. A 100, 333 (2010).

${ }^{7}$ P. Alonso-Gonzalez, M. Schnell, P. Sarriugarte, H. Sobhani, C. Wu, N. Arju, A. Khanikaev, F. Golmar, P. Albella, L. Arzubiaga, F. Casanova, L. E. Hueso, P. Nordlander, G. Shvets, and R. Hillenbrand, Nano Lett. 11, 3922 (2011).

${ }^{8}$ J. B. Lassiter, H. Sobhani, M. W. Knight, W. S. Mielczarek, P. Nordlander, and N. J. Halas, Nano Lett. 12, 1058 (2012).

${ }^{9}$ M. Frimmer, T. Coenen, and A. F. Koenderink, Phys. Rev. Lett. 108, 077404 (2012).

${ }^{10}$ M. Rahmani, D. Y. Lei, V. Giannini, B. Lukiyanchuk, M. Ranjbar, T. Y. F. Liew, M. Hong, and S. A. Maier, Nano Lett. 12, 2101 (2012).

${ }^{11}$ Y. Zhang, F. Wen, Y.-R. Zhen, P. Nordlander, and N. J. Halas, Proc. Natl. Acad. Sci. U.S.A. 110, 9215 (2013).

${ }^{12}$ E. Prodan, C. Radloff, N. J. Halas, and P. Nordlander, Science 302, 419 (2003).

${ }^{13}$ D. W. Brandl, N. A. Mirin, and P. Nordlander, J. Phys. Chem. B 110, 12302 (2006).

${ }^{14}$ Y. A. Urzhumov, G. Shvets, J. A. Fan, F. Capasso, D. Brandl, and P. Nordlander, Opt. Express 15, 14129 (2007).

${ }^{15}$ F. Hao, Y. Sonnefraud, P. V. Dorpe, S. A. Maier, N. J. Halas, and P. Nordlander, Nano Lett. 8, 3983 (2008).

${ }^{16}$ J. A. Fan, C. Wu, K. Bao, J. Bao, R. Bardhan, N. J. Halas, V. N. Manoharan, P. Nordlander, G. Shvets, and F. Capasso, Science 328, 1135 (2010).

${ }^{17}$ A. E. Miroshnichenko and Y. S. Kivshar, Nano Lett. 12, 6459 (2012).

${ }^{18}$ A. B. Evlyukhin, C. Reinhardt, A. Seidel, B. S. Luk'yanchuk, and B. N. Chichkov, Phys. Rev. B 82, 045404 (2010).

${ }^{19}$ A. García-Etxarri, R. Gómez-Medina, L. S. Froufe-Pérez, C. López, L. Chantada, F. Scheffold, J. Aizpurua, M. Nieto-Vesperinas, and J. J. Sáenz, Opt. Express 19, 4815 (2011).

${ }^{20}$ J. M. Geffrin, B. García-Etxarri, R. Gómez-Medina, P. Albella, L. S. Froufe-Pérez, C. Eyraud, A. Litman, R. Vaillon, F. González, M. NietoVesperinas, J. J. Sáenz, and F. Moreno, Nat. Commun. 3, 1171 (2012).

${ }^{21}$ A. I. Kuznetsov, A. E. Miroshnichenko, Y. H. Fu, J. Zhang, and B. Luk'yanchuk, Sci. Rep. 2, 492 (2012).

${ }^{22}$ A. B. Evlyukhin, S. M. Novikov, U. Zywietz, R.-L. Eriksen, C. Reinhardt, S. I. Bozhevolnyi, and B. N. Chichkov, Nano Lett. 12, 3749 (2012).

${ }^{23}$ Y. H. Fu, A. I. Kuznetsov, A. E. Miroshnichenko, Y. F. Yu, and B. Luk'yanchuk, Nat. Commun. 4, 1527 (2013).

${ }^{24}$ U. Zywietz, C. Reinhardt, A. Evlyukhin, T. Birr, and B. Chichkov, Appl. Phys. A 1 (2013).

${ }^{25}$ I. Staude, A. E. Miroshnichenko, M. Decker, N. T. Fofang, S. Liu, E. Gonzales, J. Dominguez, T. S. Luk, D. N. Neshev, I. Brener, and Y. Kivshar, ACS Nano 7, 7824 (2013).

${ }^{26}$ B. Hopkins, W. Liu, A. E. Miroshnichenko, and Y. S. Kivshar, Nanoscale 5, 6395 (2013).

${ }^{27}$ D. S. Filonov, A. E. Krasnok, A. P. Slobozhanyuk, P. V. Kapitanova, E. A. Nenasheva, Y. S. Kivshar, and P. A. Belov, Appl. Phys. Lett. 100, 201113 (2012).

${ }^{28}$ G. W. Mulholland, C. F. Bohren, and K. A. Fuller, Langmuir 10, 2533 (1994).

${ }^{29}$ B. Hopkins, A. N. Poddubny, A. E. Miroshnichenko, and Y. S. Kivshar, Phys. Rev. A 88, 053819 (2013). 